\title{
Estado epiléptico: Innovaciones ante este constante reto neurológico
}

\author{
Status epilepticus: Innovations for this constant neurological challenge \\ Elma M. Paredes-Aragón, ${ }^{a}$ Salvador Martínez-Medina, ${ }^{a}$ Enrique Gomez-Figueroa, ${ }^{\mathrm{b}}$ Iris E. Martínez-Juárez ${ }^{\mathrm{a}, 1}$ \\ ${ }^{a}$ Departamento de Epileptología Clínica, Instituto Nacional de Neurología y Neurocirugía, Ciudad de México, México \\ ${ }^{b}$ Departamento de Neurología, Instituto Nacional de Neurología y Neurocirugía, Ciudad de México, México
}

Historia del artículo:

Recibido: 2 diciembre 2019

Revisado: 11 diciembre 2019

Aceptado: 11 diciembre 2019

Publicado 30 diciembre 2019

Palabras clave

Clasificación

Estado epiléptico

Etiología

Pronostico

Tratamiento

Keywords

Classification

Etiology

Prognosis

Status epilepticus

Treatment

\begin{abstract}
RESUMEN. Introducción: El estado epiléptico (EE) es una urgencia neurológica de gran impacto en la morbimortalidad de los pacientes neurológicos. Métodos: Se realizó revisión de literatura utilizando como palabras clave en PUBMED y EMBASE: "estado epiléptico", combinado con los términos "tratamiento", "etiología", "pronóstico", clasificación”. Se enfatizó en publicaciones sobre avances en el tema. Desarrollo: Se ha evolucionado en conocimiento y conceptos relacionados al diagnóstico y mecanismos del EE, las guías de tratamiento indican tratamiento intensivo más prontamente. Se conoce más de las causas, pero los avances en tratamiento son modestos y el pronóstico sigue siendo complejo y con alta mortalidad. El tratamiento para EE es tiempo-dependiente por su fisiopatología, pero la insuficiente preparación del personal que atiende los casos y la limitación de acceso a fármacos empeora el pronóstico. Conclusión: Aunque la investigación debe continuar para entender más los mecanismos del EE y encontrar nuevos tratamientos, el diagnóstico adecuado, el pronto tratamiento y cuando sea posible la prevención, siguen siendo la clave para mejorar el pronóstico de los pacientes con EE.
\end{abstract}

\begin{abstract}
Introduction: Status epilepticus (SE) is a neurological emergency with great impact on morbidity and mortality of neurological patients. Methods: Literature review was carried out using as keywords for PUBMED and EMBASE: "status epilepticus", combining the terms "treatment", "etiology", "prognosis", classification". Emphasis was placed on publications on advances in the subject. Results: Knowledge and concepts related to diagnosis and mechanisms of SE have evolved, treatment guidelines indicate intensive treatment more quickly. Most causes are known, but the advances in treatment are modest, and the prognosis is still complex and includes high mortality. The treatment for SE is time-dependent due to its pathophysiology, but the insufficient preparation of personnel treating the cases and the limitations in access to drugs worsens the prognosis. Conclusion: Although research must continue to better understand the mechanisms of SE to find new treatments, proper diagnosis, prompt treatment and prevention when possible, remain as key strategies to improve the prognosis of patients with SE.
\end{abstract}

\section{Introducción}

La epilepsia es el trastorno neurológico más frecuente de la neurología. El estado epiléptico (EE) es una entidad clínica común en centros de segundo y tercer nivel de atención de urgencias médicas y neurológicas. Se define como una crisis clínica que dure más de 5 minutos o crisis continuas sin recuperación de la consciencia entre episodios. Es una urgencia neurológica e incluso en un comunicado establecido por la Liga Internacional de la Epilepsia
(International League Against Epilepsy, por sus siglas ILAE en inglés), se estableció que la frase de "tiempo es cerebro" acuñada a eventos vasculares cerebrales también debe ser aplicada al estado epiléptico, debido a sus secuelas de muerte neuronal, generación de circuitos anómalos y zonas de gliosis o esclerosis mesial temporal.

El EE se manifiesta como síndromes distintos, cada uno con definición clínica y electroencefalográfica. De la misma forma en la que se establece el diagnóstico se debe realizar una investigación minuciosa de etiología subyacente y

\footnotetext{
${ }^{1}$ Autor corresponsal: irisepineuro@gmail.com. Depto. de Epileptología Clínica, Instituto Nacional de Neurología y Neurocirugía, Av. Insurgentes Sur, \#3877 CP 14269, Ciudad de México, México 
clasificación del subtipo de EE, puesto que estos factores determinan el pronóstico y tratamiento. Esta revisión bibliográfica se enfocó que las novedades para el tratamiento, diagnóstico y pronóstico de este ancestral y constante reto neurológico.

\section{Métodos}

Se hizo una revisión de palabras clave en PUBMED y EMBASE, "estado epiléptico", "tratamiento del estado epiléptico“, "etiología del estado epiléptico"., "pronóstico del estado epiléptico“, clasificación estado epiléptico“. Se revisó especialmente el surgimiento de novedades relevantes para la práctica médica.

\section{Desarrollo}

\section{Visión desde el modelo diagnóstico 1: Definición de EE a partir de su fisiopatología}

Inicialmente, Meldrum et al. (1973), sugirieron en estudios en animales, que el estado epiléptico se definía por su temporalidad, tras la presencia de 30 minutos de crisis epilépticas motoras (antes convulsivas) que llevaban a muerte neuronal (Meldrum, Vigouroux, \& Brierley, 1973). Este concepto fue modificado por Lowenstein et al., trás la preocupación del empeoramiento pronóstico al pasar mucho tiempo durante una crisis; entonces, se determinó que el estado epiléptico generalizado en el adulto y niños mayores de 5 años se diagnosticaba operacionalmente cuando: habían más de 5 minutos de crisis continuas, o cuando habían 2 o más crisis discretas entre las cuales no había completo regreso a consciencia (Trinka et al., 2015).

Trinka et al. propusieron en 2015 la definición conceptual del EE así: "El estado epiléptico es una condición que resulta de fallo de los mecanismos responsables de término de crisisa. o de inicio de los mecanismos que llevan a crisis anormalmente prolongadas" (después del punto temporal T1) (Trinka et al., 2015). Es una condición que puede tener consecuencias a largo plazo (después del punto temporal T2) que incluyen muerte neuronal, daño neuronal, alteración de circuitos neuronales dependiendo del tipo y duración de las crisis.. T1 se refiere a la duración de la crisis que es más prolongada de lo usual. T2 se refiere al tiempo de actividad ictal después del tiempo cuando hay riesgo de consecuencias a largo plazo (Trinka et al., 2015). Estas dos dimensiones temporales fueron descritas con hallazgos de investigación y también con estadística clínica en la cual se pretendía establecer un valor funcional a cada una (Trinka et al., 2015; Epilepsy Foundation, 1993):

a) T1 determina el tiempo en el cual el tratamiento debe ser considerado o iniciado; el tiempo de crisis transcurridos para diagnosticarlo se estableció en 5 minutos (la mayoría de las crisis con actividad epiléptica en EEG duran menos de 5 minutos)

b) T2 determina que tan agresivo debe ser el tratamiento para prevenir consecuencias a largo plazo. El tiempo de corte se estableció en 30 minutos.

No hay evidencia contundente en el establecimiento de esta temporalidad. Estos conceptos fueron redactados con evidencia contradictoria, puesto que múltiples factores intervienen en el grado de daño neuronal además de la temporalidad (edad, tipo de crisis, intensidad del foco epiléptico y localización del foco) (Towne et al., 1994).

Se sabe que las crisis repetitivas en el EE reducen la inhibición de sistemas claves como el gabaérgico (GABAA), el cual está bien documentado (Scott, Surtees, \& Neville, 1998). Esta inhibición disminuida puede comprometer los mecanismos de terminación de una crisis. Aún no se entienden bien los mecanismos que inician crisis anormalmente prolongadas (Sánchez Fernández, Goodkin, $\&$ Scott, 2019). Dabrowska y et al. sugieren que la transmisión sostenida mediada por AMPAR (Receptor Alfa amino 3 hidroxil 5 metil 4 isoxacolepropiónico) y que las neuronas piramidales hipocampales tienen mayor reactivada durante crisis y EE (Dabrowska et al., 2019).

La activación de estos receptores puede potenciar AMPAR mediante la activación de inserción del neurotransmisor GluR1 (Walker, 2016). La muerte neuronal y muerte durante el EE resultan de una transmisión excitatoria excesiva. La potenciación de conexiones excitadoras recurrentes puede llevar a sincronía neuronal y propagación de crisis. Diversos estudios han mostrado que los antagonistas de AMPAR abortan las crisis de forma efectiva, sobre todo el EE. Se desconoce sobre el mecanismo preciso de transmisión de AMPAR (Kapur, 2018).

\section{Visión desde el modelo de diagnóstico 2: Definición a partir de la clasificación del EE}

Inicialmente se creó un modelo general que fue modificado en 1980. La ILAE estableció ejes diagnósticos para la investigación y elección de manejo para cada paciente con EP (Trinka et al., 2015). La última modificación de estos ejes fue propuesta por Trinka et al. en 2015, quienes establecieron cuatro ejes principales: Semiología, etiología, correlatos EEG y edad.

\section{Eje semiología (patrón clínico)}

Para la semiología se podían clasificar taxonómicamente por dos categorías: Presencia o ausencia de síntomas motores y grado de alteración de la consciencia, posteriormente en esta se describe la semiología de crisis según la clasificación de ILAE (Fisher et al., 2017). 


\section{Eje etiología (causas)}

Aquí (Hauser, 1990) se describieron dos categorías: etiología conocida (sintomática) entre las cuales había subcategorías: agudas (vasculares, intoxicaciones, encefalitis), remotas (postraumáticas, posencefalitis, posevento vascular), progresivas (tumor, Lafora, demencia), síndromes electroclínicos y la segunda categoría: desconocida (criptogénica) (Trinka, Höfler, \& Zerbs, 2012).

\section{Eje hallazgos electroencefalográficos}

Éste se basa en series de casos grandes descriptivas y consensos, que se han subdividido en determinación de una localización (generalizada o lateralizada), nombre del patrón encefalográfico (descargas periódicas, delta rítmico o puntaonda), morfología (ondas, número de fases, polaridad de la onda, características relacionadas con el tiempo (prevalencia, frecuencia, duración, índice de inicio y dinámica), modulación (inducido por estímulos o espontáneo) y finalmente efectos de medicación en el EEG (electroencefalograma) (Hesdorffer, Logroscino, Cascino, Annegers, \& Hauser, 1998).

\section{Eje edad}

Los casos se dividen en: neonatal (0-30 días), infancia (1 mes a 2 años), niñez (más de 2 años a 12 años), adolescencia y adultez (12 a 59 años) y adulto mayor (más de 60 años). Se describen también características de EEG según grupo edad con síndromes electro-clínicos (Trinka et al., 2015).

\section{Evolución de las guías de manejo del EE}

Aunque se conoce más de sus causas y mecanismos, el tratamiento del EE no ha sufrido mayores innovaciones, la cantidad de fármacos disponibles no ha aumentado mucho y sigue siendo un reto para los médicos en los servicios de emergencia. Actualmente, las guías para estado epiléptico sugeridas por la ILAE y que se siguen con mayor frecuencia a nivel mundial son las propuestas por la Academia Americana de Neurología en 2016 (Glauser et al., 2016). Debe insistirse en capacitar a los que atienden pacientes con EE sobre las intervenciones de acuerdo al tiempo que el paciente tiene de presentar crisis.

\section{O a 5 minutos}

Los expertos establecieron un esquema de fácil uso en el cual asignaron 0 a 5 minutos para la estabilización prehospitalaria, urgencias o el cuidado primario del paciente. Durante el mismo se debe: Estabilizar al paciente hemodinámicamente, asegurar la vía aérea y hacer el examen neurológico, vigilar signos vitales, colocar oxígeno en caso de ser necesario, tomar glucometría capilar y administrar dextrosa y tiamina intravenosa (Silbergleit et al., 2012). En este momento también se debe intentar canalización de la vía periférica, así como de ser posible toma de muestras para evaluar electrolitos séricos, hematología, toxicología y niveles de fármacos antiepilépticos.

\section{5 a 20 minutos}

Si la crisis continua, se dio un parámetro de 5 a 20 minutos para iniciar tratamiento endovenoso. La benzodiazepina es la medicación inicial de elección con un nivel A de evidencia, encontrándose en primera línea:

a) Midazolam intramuscular $(10 \mathrm{mg} / \mathrm{kg}$ en mayores de $40 \mathrm{~kg}$ en una sola dosis),

b) Lorazepam intravenoso $(0.1 \mathrm{mg} / \mathrm{kg} / \mathrm{dosis}$, pudiendo repetir una dosis adicional),

c) Diazepam intravenoso $(0.15-0.2 \mathrm{mg} / \mathrm{kg} /$ dosis, máximo $10 \mathrm{mg}$ por dosis puede repetir la dosis en una ocasión) (Alldredge et al, 2001).

\section{0 a 40 minutos}

Si la crisis persiste, para el manejo no hay evidencia que prefiera un fármaco sobre otro (Leppik, 1983). Se recomienda uno de los siguientes fármacos de segunda línea:

a) Fosfenitoína intravenoso (20mg / kg máximo 1500mg dosis máxima, se recomienda única dosis),

b) Ácido valproico intravenoso $(40 \mathrm{mg} / \mathrm{k}$, dosis máxima $3000 \mathrm{mg} /$ dosis, dosis única),

c) Levetiracetam intravenoso $60 \mathrm{mg} / \mathrm{kg}$, máximo 4500mg/dosis, dosis única) (Misra, Kalita \& Maurya, 2012; Misra, Kalita \& Patel, 2006; Gilad et al, 2008).

\section{0 a 60 minutos}

Si la crisis continua, se pasa a medicación de tercera línea en donde no hay evidencia clara para guiar la terapéutica en esta fase. Las opciones a escoger incluyen repetición de los medicamentos de segunda línea, anestésicos como tiopental, midazolam, fentobarbital o propofol (considerando su disponibilidad en la región). Todas estas deben tener monitoreo EEG continuo (Brophy et al., 2012).

\section{Sin evidencia de superioridad con los nuevos fármacos}

\section{Fenitoína}

Este es el medicamento utilizado en algunos países donde no hay fosfenitoína para el EE. La fenitoína intravenosa se ha asociado a complicaciones hemodinámicas fatales y a 
reacciones fatales en el sitio de inyección que incluye necrosis y amputación de las extremidades.

\section{Fosfenitoína}

La fosfenitoína es una prodroga de la fenitoína que tiene las mismas propiedades farmacológicas que la fenitoína, pero ninguna de las complicaciones antes mencionadas. Las ventajas potenciales encontradas con la fosfenitoína no son en cuanto a su efectividad sino a su seguridad (Petit-Pedrol et al., 2014). La fosfenitoína es más costosa a corto plazo. $\mathrm{Su}$ efectividad se ha probado in vitro e in vivo, sin ser superior a su precursora fenitoína. Por este motivo, nuestro protocolo contemplará la fenitoína como fármaco antiepiléptico de elección en casos que ameritarían manejo con fosfenitoína en el esquema antes explicado (De Toledo \& Ramsay, 2000). Ver Figura 1.

\section{Brivaracetam}

Finalmente, es importante mencionar al brivaracetam (BRV) como potencial fármaco útil en el tratamiento de segunda línea en EE. Éste es un fármaco de alta afinidad ligando de sináptico de la glicoproteína $2 \mathrm{~A}$ que se relaciona estructuralmente a levetiracetam (LEV) (Kalss et al., 2018). Comparado a LEV, el BRV es mucho más afín por el ligando y por ser más lipofílico, podría tener mayor penetración a través de la barrera hematoencefálica y potencialmente tendría mayor efecto antiepiléptico. En un estudio encabezado por Trinka y et al., se administró BRV endovenoso a pacientes con EE desde enero de 2016 a Julio de 2017. Se administró BRV tras usar cuatro fármacos en promedio. La dosis media de administración fue de 100mg en 15 minutos. Se observó mejoría en la escala de coma de Glasgow en $86 \%$ de los pacientes revisados. No se vieron efectos adversos (Kalss et al., 2018).

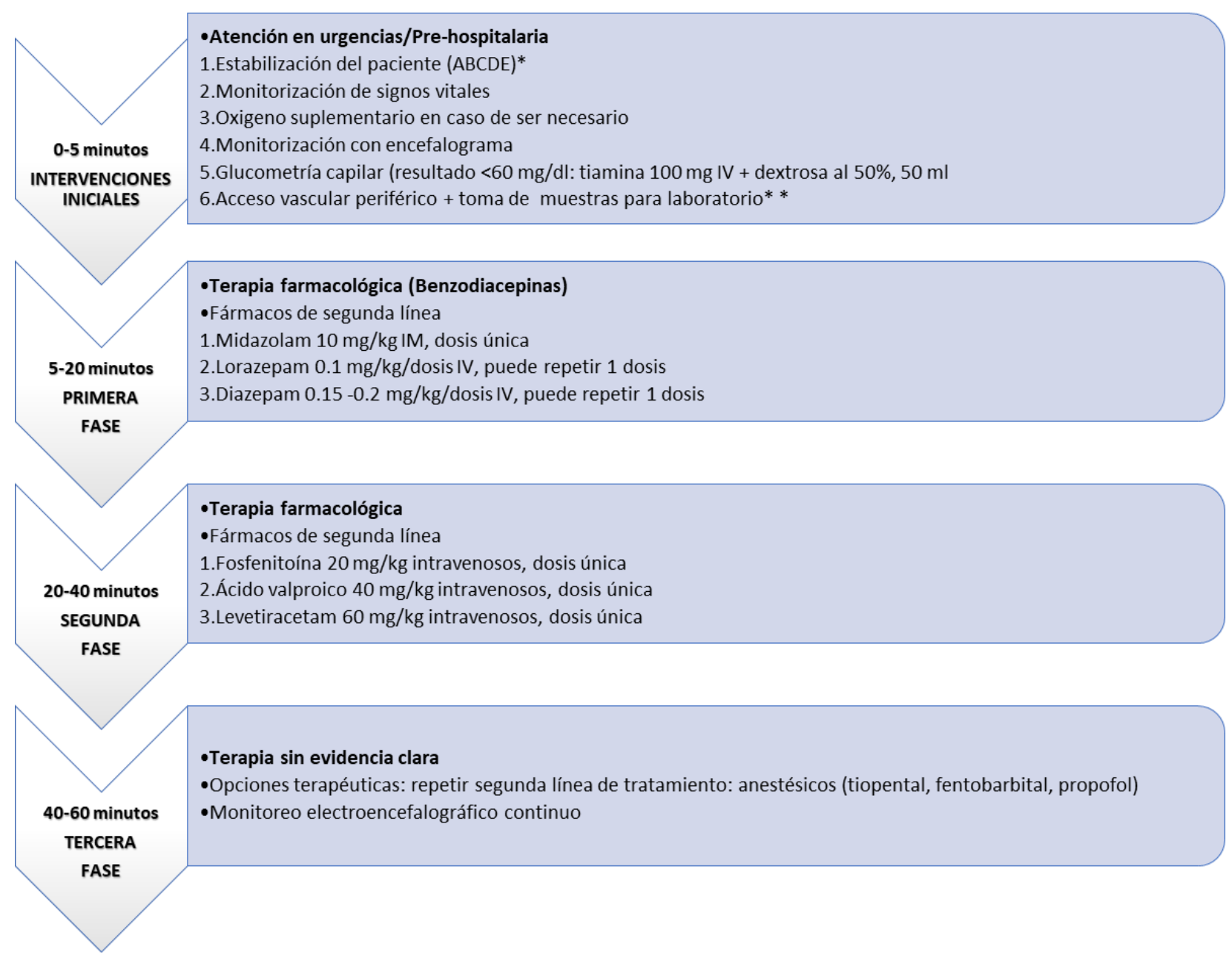

*ABCDE: Vía área, Respiración, Circulación, Déficit Neurológico, Examen general

**Laboratorio: Electrólitos séricos, biometría hemática, perfil toxicológico, niveles de fármacos

Figura 1. Algoritmo de abordaje y manejo de estado epiléptico (Academia Americana de Neurología, 2016). 


\section{Discusión}

En general, no hay avances significativos en la prevención de la morbimortalidad o mejoramiento del pronóstico del EP. Se ha reportado una incidencia anual de EE en un rango de 7 a 41 casos por cada 100,000 habitantes (Leitinger, 2019). La mayoría de los estudios basados en población han definido EE utilizando una duración de 30 minutos.

El gran rango de incidencia es por diferencias en el establecimiento de la definición de caso y en las poblaciones estudiadas. Algunos estudios incluyen EE convulsivo y no convulsivo y algunas tras excluyen EP no convulsivo. En el caso de EE convulsivo la incidencia está reportada en 7 por cada 100,000 (Sutter, Rüegg, \& Kaplan, 2012).

La incidencia del EE sigue una distribución en forma de $\mathrm{U}$, con incidencia relativamente más alta en niños con menos de un año de edad y que después aumenta en adultos mayores después de los 60 años. Durante la vida, hasta el $10 \%$ de los adultos con epilepsia y el $20 \%$ de los niños con epilepsia tendrán un episodio o más de EE (Sánchez \& Rincón, 2016).

La epilepsia refractaria es una condición potencialmente fatal, en la cual la mortalidad va desde $24-38 \%$ en series recientes, que se eleva cuando las crisis se prolongan. Una definición aceptada de EER (estado epiléptico refractario) es una crisis que persiste después de dos fármacos antiepilépticos, típicamente incluyendo un benzodiacepina.

En esta etapa, la mayoría de los protocolos sugieren tratamiento con fármacos anestésicos para frenar de forma oportuna la actividad epiléptica y prevenir daño neuronal a largo plazo, así como refractariedad consecuente y consecuencias sistémicas. La mayoría de la evidencia establecida en el manejo de EER se basa en series pequeñas, por ser menos frecuente (Kwan et al., 2010).

La etiología subyacente se considera el factor pronóstico más importante para determinar el desenlace vital del EE. A partir del tratamiento, la etiología difiere de forma significativa en países en desarrollo comparado con el mundo occidental. Las etiologías sintomáticas agudas son más frecuentes en países en desarrollo. En un estudio encabezado por Ferlisi et al. (2014), se realizó un análisis de 776 casos de EER en 50 países en 4 años. El control de estado epiléptico se logró en $74 \%$ de los casos. Los desenlaces neurológicos fueron malos: 24 a $41 \%$ de los pacientes murieron.

El buen desenlace se correlacionó con menor edad y una historia previa de epilepsia. La etiología correlacionó importantemente con la causa. Los pacientes de Asia eran más jóvenes y las etiologías eran más infecciosas que en Europa y América. A pesar de estas diferencias, los hallazgos eran similares en todos los países (Nandhagopal, 2006).
Las etiologías principales para América en orden de frecuencia fueron: desconocida/ criptogénica (22.8\%), infecciosas $(15.4 \%)$, vascular $(11.4 \%)$ y anoxia $(11.4 \%)$, reducción o suspensión de tratamiento antiepiléptico $(9.4 \%)$, infecciones fuera del sistema nervioso $(8.7 \%)$, metabólicas $(4.7 \%)$, encefalitis agudas $(4.7 \%)$, trauma (4.7\%) seguidas de etilismo, tumor cerebral, inmunológicas, meningitis aguda, toxinas, misceláneas (eclampsia, síndrome de encefalopatía posterior reversible, displasia cortical) y finalmente, genética/ cromosómica (Trinka, Höfler, \& Zerbs, 2012; Neligan \& Shorvon, 2010).

Existen muchos retos en el tratamiento de los pacientes con EE. Una causa frecuentemente asociada con mayor refractariedad y aumento de mortalidad es la falta de preparación en el manejo de EE. La educación médica continua debe combatir la falta de entrenamiento y el personal de los centros de primer y segundo nivel deben mejorar en el reconocimiento de una crisis epiléptica, en el manejo de EE y en el conocimiento de tiempos y dosis de fármacos a indicarse.

El primer reto operativo es la disponibilidad de los medicamentos en los servicios de urgencias, segundo es la correcta elección del fármaco antiepiléptico adecuado a la dosis adecuada para tratar un EE. No debe olvidarse que se debe escoger el fármaco que no precipite otras crisis que tenga el paciente (mioclonías por bloqueadores de canales de sodio, crisis tónicas por benzodiacepinas, etc.) Además, debe vigilarse y prevenirse que no se presenten efectos secundarios de algunos fármacos.

Existen epilepsias refractarias a fármacos que constituyen un reto para la preservación de la función neuronal y para el control de los circuitos excitadores corticales, que no se lograron suprimir con fármacos de primera y segunda línea. El principal motivo para esto último es la internalización de los receptores GABA o la resistencia de receptores AMPA a los fármacos antiepilépticos, entre otros. Algunos autores han propuesto que algunos pacientes son candidatos a tratamiento con segunda línea o hasta tercera línea por refractariedad de sus crisis. Finalmente, en un estudio realizado por Sánchez Fernández et al., se encontró que el inicio de EER intermitente y extrahospitalario se asocia de forma independiente con un retraso en la administración de BZD de primera línea y fármacos de la segunda línea de tratamiento. Estos factores identifican potenciales puntos de mejora para manejo de pacientes pediátricos en el tercer nivel de atención (Sánchez Fernández, Goodkin, \& Scott, 2019)

\section{Conclusión}

El mayor conocimiento del clínico sobre el EE mejorará la morbimortalidad de los pacientes que lo presentan y 
contribuirá al manejo oportuno de epilepsias que responden a primera línea de tratamiento. Es de suma importancia que se conozca el manejo del EE, desde su instancia extrahospitalaria hasta el manejo los casos refractarios. Aunque la investigación debe continuar para entender más los mecanismos del EE, la prevención en los casos que sea posible y el pronto tratamiento siguen siendo la clave para mejorar el pronóstico de los pacientes afectados con EE.

\section{Contribuciones de los Autores}

Todos los autores participaron en la revisión de la literatura incluida y redactaron el manuscrito.

\section{Conflictos de Interés}

Los autores declaran no tener ningún conflicto de interés.

\section{Referencias Bibliográficas}

Alldredge, B.K., Gelb, A.M., Marshal Isaacs, S., Corry, M.D., Allen, F., Ulrich, S., Gottwald, M.D., O`Neil, N., Neuhaus, J.M., Segal, M.R., \& Lowenstein, D.H. (2001). A comparison of lorazepam, diazepam, and placebo for the treatment of out-of-hospital status epilepticus. $N$ Engl J Med, 345, p. 631-637.

Brophy, G.M., Bell, R., Claassen, J., Alldredge, B., Bleck, T.P., Glauser, T., LaRoche, S.M., Riviello, J.J., Shutter, L., Sperling, M.R., Treiman, D.M., Vespa, P.M., \& Neurocritical Care Society Status Epilepticus Guideline Writing Committee. (2012). Guidelines for the evaluation and management of status epilepticus. Neurocrit Care, 17, p. 3-23.

Dabrowska, N., Joshi, S., Williamson, J., Lewczuk, E., Lu, Y., Oberoi, S., Brodovskaya A., \& Kapur, J. (2019). Parallel pathways of seizure generalization. Brain, 142, p. 2336-2351.

DeToledo, J.C., \& Ramsay, R.E. (2000). Fosphenytoin and phenytoin in patients with status epilepticus. Improved tolerability versus increased costs. Drug Saf, 22, p. 459-466.

Ferlisi, M., \& Shorvon, S. (2014). Seizure precipants (triggering factors) in patients with epilepsy. Epilepsy Behav., 33, p. 101-105.

Fisher, R.S., Helen Cross, J., French, J.A., Higurashi, N., Hirsch, E., Jansen, F.E., Lagae, L., Moshé, S.L., Peltola, J., Roulet Perez, E., Scheffer, I.E., \& Zuberi, S.M. (2017). Operational classification of seizure types by the International League Against Epilepsy: Position Paper of the ILAE Commission for Classification and Terminology. Epilepsia, 58, p. 522530.

Gilad, R., Izkovitz, N., Dabby, R., Rapoport, A., Sadeh, M., Weller, B., \& Lampl, Y. (2008). Treatment of status epilepticus and acute repetitive seizures with i.v. valproic acid vs phenytoin. Acta Neurol Scand, 118, p. 296-300.

Glauser, T., Shinnar, S., Gloss, D., Alldredge, B., Arya, R., Bainbridge, J., Bare, M., Bleck, T., Dodson E.W., Garrity, L., Jogoda, A., Lowenstein, D., Pellock, J., Riviello, J., Sloan, E., \& Treiman, D.M. (2016). Evidence-based guideline: Treatment of convulsive status epilepticus in children and adults: Report of the guideline committee of the American epilepsy society. Epilepsy Curr, 16, p. 48-61.
Hauser, W.A. (1990). Status epilepticus: epidemiologic considerations. Neurology, 40, p. 9-13.

Hesdorffer, D.C., Logroscino, G., Cascino, G., Annegers, J.F., \& Hauser, W.A. (1998). Incidence of status epilepticus in Rochester, Minnesota, 1965-1984. Neurology, 50, p.735-741.

Kapur, J. (2018). Role of NMDA receptors in the pathophysiology and treatment of status epilepticus. Epilepsia Open, 3, p.165-168.

Kalss, G., Rohracher, A., Leitinger, M., Pilz, G., Novak, H.F., Neuray, C., Kreidenhuber, R., Höfler, J., Kuchukhidze, G., \& Trinka, E. (2018). Intravenous brivaracetam in status epilepticus: A retrospective singlecenter study. Epilepsia, 59, p.228-233.

Kwan, P., Arzimanoglou, A., Berg, A.T., Brodie, M.J., Hauser, W.A., Mathern, G., Moshé, S.L., Perucca, E., Wiebe, S., \& French, J. (2010). Definition of drug resistant epilepsy: Consensus proposal by the ad hoc Task Force of the ILAE Commission on Therapeutic Strategies. Epilepsia, 51, p.1069-1077.

Leitinger, M., Trinka, E., Giovannini, G., Zimmermann, G., Florea, C., Rohracher, A., Kalss, G., Neuray, C., Kreidenhuber, R., Höfler, J., Kuchukhidze, G., Granbichler, C., Dobesberger, J., Novak, H.F., Pilz, G., Meletti, S., \& Siebert, U. (2019). Epidemiology of status epilepticus in adults: A population-based study on incidence, causes, and outcomes. Epilepsia, 60, p. 53-62.

Leppik, I.E., Derivan, A.T., Homan, R.W., Walker, J., Ramsay, R.E. \& Patrick, B. (1983). Double-blind study of lorazepam and diazepam in status epilepticus. JAMA, 249, p.1452-1454.

Meldrum, B.S., Vigouroux, R.A., \& Brierley, J.B. (1973). Systemic Factors and Epileptic Brain Damage: Prolonged Seizures in Paralyzed, Artificially Ventilated Baboons. Arch Neurol, 29, p. 82-87.

Misra, U.K., Kalita, J., \& Maurya, P.K. (2012). Levetiracetam versus lorazepam in status epilepticus: A randomized, open labeled pilot study. J Neurol; 259, p. 645-648.

Misra, U.K., Kalita, J., \& Patel, R. (2006) Sodium valproate vs phenytoin in status epilepticus: A pilot study. Neurology, 67, p. 340-342.

Nandhagopal, R. (2006). Generalised convulsive status epilepticus: An overview. Postgrad Med J, 82, p. 723-372.

Neligan, A. \& Shorvon S.D. (2010). Frequency and prognosis of convulsive status epilepticus of different causes: A systematic review. Arch Neurol, 67, p. 931-940.

Petit-Pedrol, M., Armangue, T., Peng, X., Bataller, L., Cellucci, T., Davis, R., McCracken, L., Martinez-Hernandez, E., Mason, W.P., Kruer, M.C., Ritacco, D.G., Grisold, W., Meaney, B.F., Alcalá, C., SillevisSmitt, P., Titulaer, M.J., Balice-Gordon, R., Graus, F., \& Dalmau, J. (2014). Encephalitis with refractory seizures, status epilepticus, and antibodies to the GABA A receptor: A case series, characterisation of the antigen, and analysis of the effects of antibodies. Lancet Neurol, 13, p. 276-286.

Sánchez Fernández, I., Goodkin, H.P., \& Scott, R.C. (2019). Pathophysiology of convulsive status epilepticus. Seizure; 68: p.16-21.

Sánchez, S. \& Rincon, F. (2016). Status Epilepticus: Epidemiology and Public Health Needs. J Clin Med, 5:71.

Scott, R.C., Surtees, R.A.H., \& Neville, B.G.R. (1998). Status epilepticus: Pathophysiology, epidemiology, and outcomes. Arch Dis Child, 79, p. 73-77. 
Silbergleit, R., Durkalski, V., Lowenstein, D., Conwit, R., Pancioli, A., Palesch, Y., Barsan, W., \& NETT Investigators. (2012). Intramuscular versus intravenous therapy for prehospital status epilepticus. $N$ Engl J Med, 366, p. 591-600.

Strzelczyk, A., Zöllner, J.P., Willems, L.M., Jost, J., Paule, E., SchubertBast, S., Rosenow, F., \& Bauer, S. (2017). Lacosamide in status epilepticus: Systematic review of current evidence. Epilepsia, 58, p. 933-950.

Sutter, R., Rüegg, S., \& Kaplan, P.W. (2012). Epidemiology, diagnosis, and management of nonconvulsive status epilepticus: Opening Pandora's box. Neurol Clin Pract, 2, p. 275-286.

Towne, A.R., Pellock, J.M., Ko, D., \& DeLorenzo, R.J. (1994). Determinants of mortality in status epilepticus. Epilepsia, 35, p. 27-34. Trinka, E., Cock, H., Hesdorffer, D., Rossetti, A.O., Scheffer, I.E., Shinnar, S., Shorvon, S., \& Lowenstein, D.H. (2015). A definition and classification of status epilepticus - Report of the ILAE Task Force on Classification of Status Epilepticus. Epilepsia, 56, p.1515-1523.

Trinka, E., Höfler, J., \& Zerbs A. (2012). Causes of status epilepticus. Epilepsia, 53, p.127-138.

Epilepsy Foundation. (1993). Treatment of Convulsive Status Epilepticus: Recommendations of the Epilepsy Foundation of America's Working Group on Status Epilepticus. JAMA, 270, p.854-859.

Treiman, D.M., Meyers, P.D., Walton, N.Y., Collins, J.F., Colling, C., Rowan, A.J., Handforth, A., Faught, E., Calabrese, V.P., Uthman, B.M., Ramsay, R.E., \& Mamdani, M.B. (1998). A comparison of four treatments for generalized convulsive status epilepticus. $N$ Engl J Med, 339, p. 792-798.

Walker, M.C. (2018). Pathophysiology of status epilepticus. Neurosci Lett, 667, p. 84-91 